Reprod. Nutr. Dévelop., 1986, 26 (1 B), 327-328.

\title{
Importance et lieu de la digestion des polyosides des parois cellulaires d'un foin de graminée chez le mouton
}

\author{
Sylvie POCHET
}

Laboratoire de la Digestion des Ruminants, I.N.R.A. Theix, 63122 Ceyrat, France.

Summary. The purpose of this work was to determine the fate of cell wall polysaccharides from a graminaceous hay in the digestive tract of sheep. Emphasis was placed on determining the true digestibility of each polysaccharide in the stomachs.

L'objectif de ce travail a été d'étudier le devenir des différents oses constitutifs des hémicelluloses et de la cellulose du foin de dactyle au cours du transit digestif chez le mouton, et d'évaluer plus particulièrement l'utilisation digestive réelle de ces polyosides dans les réservoirs gastriques.

Matériel et méthodes. Six moutons de race Texel d'environ $45 \mathrm{~kg}$, munis d'une canule du rumen et d'une canule simple du duodénum proximal, ont reçu un régime de foin de dactyle (récolté à l'épiaison du 1er cycle) distribué sous forme hachée, en deux repas égaux, et ingéré à raison de $55 \mathrm{~g}$ de matière sèche par $\mathrm{kg}$ de $\mathrm{P}^{0,75}$. L'oxyde de chrome sur support de cellulose a été utilisé comme marqueur du transit. Après une période de mesure de la digestibilité des constituants de la ration, on a prélevé pour chaque animal un échantillon représentatif du contenu digestif transitant en $24 \mathrm{~h}$ au niveau duodénal, obtenu par cumul des fractions de $100 \mathrm{ml}$ prélevées à intervalle de $6 \mathrm{~h}$ pendant 3 jours consécutifs. Les échantillons d'aliment et de fèces ont été séchés et les contenus duodénaux lyophilisés. Tous les échantillons secs ont été broyés à la grille de $0,8 \mathrm{~mm}$.

L'isolement et le fractionnement des parois cellulaires a été réalisé par la méthode de Jarrige (1961) semi-automatisée sur Fibertec (Pochet, à paraître). Les teneurs en hémicelluloses et cellulose ont été estimées par dosage du pouvoir réducteur des hydrolysats acides par la méthode d'Hoffman automatisée (1966). La composition de ces deux fractions a été déterminée par chromatographie liquide sur résine échangeuse d'ions par les oses neutres (Besle, 1974), et par réaction au MHDP pour les acides uroniques (Blumenkrantz et Asboe-Hansen, 1973). Le flux d'azote bactérien à l'entrée de l'intestin grêle a été estimé par le rapport des concentrations d'acide - diaminopimélique des contenus de duodénum et des bactéries isolées du jus de rumen des mêmes animaux par centrifugation fractionnée.

Résultats et discussion. En accord avec les résultats obtenus par Jarrige et Minson (1964), les xylanes qui représentent les $2 / 3$ des hémicelluloses du dactyle et plus de $85 \%$ des résidus hémicellulosiques des fèces, sont responsables de la moins bonne utilisation digestive des hémicelluloses par rapport à celle de la cellulose ; la digestibilité des oses hémicellulosiques autres que le xylose est toutefois importante, de $79 \%$ à $84 \%$ pour le galactose, l'arabinose et les acides uroni- 
ques, à près de $90 \%$ pour le glucose et supérieure donc à celle observée pour les oses cellulosiques (68 à $78 \%$ ) (tabl. 1). Dans les réservoirs gastriques disparaissent plus de $85 \%$ des polyosides digestibles de la paroi. Cette proportion est plus faible pour les hémicelluloses que pour la cellulose. Hormis l'arabinose et les acides uroniques qui sont dégradés de façon aussi importante que la fraction cellulose $(90 \%)$, une partie importante du xylose $(16 \%)$, du galactose $(20 \%)$ et du glucose $(30 \%)$ des hémicelluloses semble échapper à l'action du rumen. Utilisant les résultats de Mc Allan et Smith (1974) qui estiment à $25 \mathrm{~g} / \mathrm{kg}$ de matière sèche la teneur en glucose des bactéries isolées dans le cas d'un régime foin, nous avons calculé à partir du flux d'azote bactérien, que 50 à $95 \%$ des glucanes isolés dans la fraction hémicellulosique qui sort des estomacs proviennent en fait des polyosides bactériens. Cette contamination conduit à une sous-estimation des quantités de glucose hémicellulosique digestible disparaissant réellement dans le rumen, qui varient en fait de 90 à $102 \%$ de l'ingéré. Une correction identique portant sur les autres oses constitutifs des polyosides bactériens des contenus de duodénum et des fèces conduit à une augmentation de l'utilisation digestive réelle des polyosides pariétaux, notamment des hémicelluloses, dans le tractus digestif entier et dans les estomacs en particulier.

TABL. 1. - Composition et utilisation digestive des polyosides pariétaux du foin de dactyle.

\begin{tabular}{lccc}
\hline & $\begin{array}{c}\text { Composition } \\
\text { du foin } \\
(\% \mathrm{M} . \mathrm{S} .)\end{array}$ & $\begin{array}{c}\text { Digestibilité } \\
(\mathrm{n}=6) \\
(\% \text { ingéré) }\end{array}$ & $\begin{array}{c}\text { Digéré dans les } \\
\text { estomacs }(\mathrm{n}=3) \\
(\% \text { digestible })\end{array}$ \\
\hline Matière organique & 88,0 & $62,6(1,3)$ & $65,8(4,1)$ \\
Hémicelluloses & 14,8 & $67,6(1,4)$ & $82,2(1,7)$ \\
- Acides uroniques & 2,7 & $83,6(2,6)$ & $92,6(5,9)$ \\
- Arabinose & $19,4\left(^{*}\right)$ & $83,1(1,8)$ & $91,2(1,9)$ \\
- Galactose & $4,5\left(^{*}\right)$ & $78,9(3,1)$ & $80,5(6,9)$ \\
- Xylose & $66,7\left(^{*}\right)$ & $59,2(1,9)$ & $83,5(3,1)$ \\
- Glucose & $9,4\left(^{*}\right)$ & $89,5(2,1)$ & $71,3(7,0)$ \\
$\quad$ Pentoses $\quad$ Hexoses & $86,1\left(^{*}\right)$ & $64,6(1,8)$ & $84,2(1,9)$ \\
Cellulose & $13,9\left(^{*}\right)$ & $86,0(2,3)$ & $74,0(6,7)$ \\
- Xylose & 21,9 & $76,7(1,1)$ & $89,2(1,4)$ \\
- Glucose + cellobiose & $8,4\left(^{*}\right)$ & $68,0(3,6)$ & $96,1(1,4)$ \\
Hemicellulose + cellulose & $91,6\left(^{*}\right)$ & $77,6(1,0)$ & $88,8(1,6)$ \\
\hline
\end{tabular}

$\left(^{*}\right)$ Résultats exprimés en pour cent du mélange des oses détectés.

Besle J. M., 1974. Séparation des oses, des di- et triolosides par chromatographie d'échange d'ions. Ann. Biol. anim. Bioch. Biophys., 14, 545-573.

Blumenkrantz N., Asboe-Hansen G., 1973. New method for quantitative determination of uronic acids. Analyst. Bioch., 54, 484-489.

Hoffman, 1966. Dosage automatique du glucose. Technicon méthodologie $2 \mathrm{~B}$.

Jarrige R., 1961. Analyse des constituants glucidiques des plantes fourragères (1). Ann. Biol. anim. Bioch. Biophys., 1, 163-212.

Jarrige R., Minson D. J., 1964. Digestibilité des constituants du ray-grass anglais 524 et du dactyle S37 plus généralement des constituants glucidiques. Ann. Zoot., 13, 117-150.

McAllan A. B., Smith R. H., 1974. Carbohydrate metabolism in the ruminant. Br. J. Nutr., 31, 77-88. 